\title{
Comparative Study between Conventional Methods over Automated Methods for Speciation of Coagulase Negative Staphylococcus in a Tertiary Care Center
}

\author{
S.S.M. Umamagewari ${ }^{1 *}$, P. Neelu Sree ${ }^{1}$, Madhurima Mukerjee ${ }^{2}$, \\ M. Kalyani ${ }^{1}$ and A.S. Shameem banu ${ }^{2}$ \\ ${ }^{1}$ Department of Microbiology, Saveetha Medical College and Hospital, \\ Thandalam, Chennai, India \\ ${ }^{2}$ Chettinad Medical College, Chennai, India \\ *Corresponding author
}

Keywords

CONS,

Methicillin resistant CONS, Staphylococcus hemolyticus.

\section{Article Info}

Accepted:

20 September 2016

Available Online:

10 October 2016
A B S T R A C T

Coagulase negative staphylococcus are the commensal of humans, which are increasingly being recognized as significant nosocomial pathogens, mainly in patients under long term medical care or in immunocompromised patients. Identification and speciation of CONS is becoming medically important due to its multidrug resistant patterns and to define its clinical significance. Study was carried out from of Dec 2014 till May 2015. A total of 60 isolates from various clinical specimen was collected in a tertiary care centre. Speciation was done by conventional method which included biochemical characterization and automated method like Vitek 2 GP 21342. Antibiotic sensitivity testing was done by Disc diffusion method on Muller Hinton Agar. Out of 60 isolates Staphylococcus haemolyticus (26\%) was most frequently isolated, followed by S.cohnii (23\%), S.xylosus (13\%), S.lugdunensis (11\%), S.epidermis (10\%), S.stimulans (10\%), S.saprophyticus (3\%), S.schleiferi (3\%) and S.warnerii (3\%). This finding was based on the conventional method of speciating CONS. From the total of 60 isolates Vitek correctly identified S.hamolyticus (26\%), s.cohnii(23\%), S.epidermidis $(10 \%)$ and S.saprophyticus (3\%), for the remaining species like S.xylosus, S.lugdunensis, S.simulans, S.schleiferi and S.warnerii the result was ambiguous and correct identification was done based on the supplemental test suggested by the manufacturer, by the conventional method only $3 \%$ was misidentified to be S.warneri which the automated method idnetified to be S.epidermidis. Antibiotic sensitivity testing showed $36 \%$ of methicillin resistant CONS and $63 \%$ of methicillin sensitive CONS from the same 60 isolates. Resistances to other group of drugs were also studied. Emergence of various drug resistances makes it important for speciation of CONS and to study their Antibiogram pattern for treating the infection caused by these organisms. Though speciation of CONS by automated method is expensive but it is reliable and accurate than conventional method which is more cumbersome and variable results are obtained. 


\section{Introduction}

Coagulase negative staphylococci (CONS) are increasingly being recognized as significant nosocomial pathogens partly due to the growing reports of this group of organism causing opportunistic infections. Though CONS is considered to be a commensal of humans, it is found to cause nosocomial infection in patient under transient or permanent medical care or in immunocompromised patients (Goyal, 2006).

CONS are one of the most frequent causes of nosocomial outbreaks in hospitals and are reservoirs of multiple antimicrobial resistant determinants. Identification and speciation of CONS has become important due to the growing recongnition and medical importance to define their clinical significance (Goyal, 2006; Shubra Singh, 2008).

Routinely isolation of genus staphylococci was done based on catalase test. For differentiating coagulase positive and coagulase negative staphylococci were done based on slide coagulase and tube coagulase test. Few commonly isolated species of Genus Staphylococcus are given in table 1 (Wesley et al., 1994).

Further speciation of CONS by reference method of Kloos and Schleifer is cumbersome, time consuming and requires expensive reagents which are not routinely available in most of the clinical laboratories. There are several manual biochemical methods for identifying CONS species. However these are based on phenotypic characters which are variable in their expression and consequently these methods have high error rates and therefore serious limitation (Wesley et al., 1975).

Many automated methods are being used to speciate CONS. Various different techniques have been developed for accurate and rapid identification of CONS. For example : PCR-RFLP, API staph method, Rapidec Staph, Microscan Pos ID Panel, BBL Crystal Gram (+) identification system, Vitek GP identification Card (Konemann).

Vitek 2 system used with the gram positive (GP) identification card (biomerieux) is an automated machine designed to provide rapid and accurate phenotypic identification for most clinical isolates and their susceptibility testing (Konemann).

The objective of the present study is to speciate CONS based on conventional method and automated method and to compare the efficacy between them in clinical laboratory.

\section{Materials and Methods}

\section{Study design}

This study is prospective study conducted in a tertiary care centre in a time period of 6 months that is from December 2014 - May 2015. The sample size of this study is 60 clinical isolates and it was collected from various clinical samples like Exudate, Blood and Urine. Transported immediately to the laboratory for investigation.

\section{Conventional method}

Strains collected were isolated as described by Konemann et al., (1997). The isolates from clinical specimens were plated onto blood agar and gram staining was performed to guarantee the purity and to study their morphologic structure. Based on the gram staining results Catalase and coagulase tests (slide and tube) were done. After confirmation of the family Micrococcaceae, Staphylococcus was differentiated from Micrococcus species on the basis of 
oxidation and fermentation test and resistance to bacitracin indicated by absence of an inhibition halo or presence of a inhibition halo measuring upto $9 \mathrm{~mm}$ in diameter (Maria et al., 2004).

Speciation of CONS was done based on reference method proposed by Kloos and Schleifer (1975) and Banermann (2003). This method consists of a set of biochemical test that determines the utilization of sugars like xylose, arabinose, sucrose, trehaolse, maltose, mannitol, lactose, ribose, fructose and mannose, production of hemolysin, nitrate reduction, presence of urease, ornithine decarboxylase and resistance to novobiocin characterized by inhibition halo of upto $16 \mathrm{~mm}$. Readings of the tests were obtained after 24,36,72 hours of incubation at $37^{\circ} \mathrm{C}$ in an air incubator. The following scheme was followed to speciate the isolate and to identify it given in table 2 and table 3 (Maria et al., 2004; Dawne, 1993).

The susceptibility of the isolates to antimicrobial agents was also determined by disk diffusion method with Mueller-Hinton plates according to the CLSI guidelines 2015-16 (Maurizio Sanguinetti, 2003). Disk containing following antibiotic at the specific absolute concentration was used: Penicillin (10units), Cefazolin $(30 \mu \mathrm{g})$, Cloxacillin $(30 \mu \mathrm{g})$, Gentamicin $(10 \mu \mathrm{g})$, Netilmycin $(30 \mu \mathrm{g})$, Ciprofloxacin $(5 \mu \mathrm{g})$, Cotrimoxazole $(1.25 \mu \mathrm{g}), \quad$ Erythromycin $(15 \mu \mathrm{g})$, Clindamycin $(2 \mu \mathrm{g})$, Tetracycline $(30 \mu \mathrm{g})$, Vancomycin $(30 \mu \mathrm{g})$, Teicoplanin $(30 \mu \mathrm{g})$ and Linezolid $(30 \mu \mathrm{g})$.

\section{Automated method}

The vitek 2 system was used according to the manufacturer's instructions, ID - Gram Positive Cocci cards (ID-GPC cards bioMerieux) were used for identification. The ID-GPC database of the VITEK 2 system includes the following taxa:
S. aureus, Staphylococcus auricularis, Staphylococcus capitis, Staphylococcus chromogenes, Staphylococcus cohnii subsp. cohnii, S. cohnii subsp. urealyticus, $S$. epidermidis, Staphylococcus haemolyticus, Staphylococcus hominis, Staphylococcus hyicus, Staphylococcus intermedius, Staphylococcus kloosii, Staphylococcus lentus, Staphylococcus lugdunensis, Staphylococcus saprophyticus, Staphylococcus schleiferi, Staphylococcus sciuri, Staphylococcus simulans, Staphylococcus warneri, and Staphylococcus xylosus (Dawne, 1993).

The ID_GPC card is a 64 - well plastic card containing 18 empty wells and 46 wells for fluroscent and inhibitory tests that include $\mathrm{pH}$ change tests and derivatives to detect aminopepetidases and osidases. Substrates used for detection of aminopeptidases are usually coupled with 7-aminomethylcoumarin (7AMC). Substrates used for detection of osidases are coupled with 4methlybelliferone (4MU) (Dawne, 1993).

The 21 tests are as follows: 4MU- $\alpha-1-$ arabinifuranoside, 4MU- $\alpha$-d-galactoside, 4MU- $\beta$-d-glucouronide, $\quad 4 M U-\alpha-d-$ glucoside, $\quad 4 \mathrm{MU}-\alpha-\mathrm{d}-\mathrm{N}$-acetylneuraminic acid, 4MU- $\beta$-d-galactoside, 4MU- $\beta-\quad \mathrm{d}-$ glucoside, 4MU- $3-d-m a n n o s i d e, \quad 4 M U-n-$ acetyl- $\beta$-d-glucosamide, 4MU-phosphate, alanine-7AMC, arginine7AMC, aurease(butiloxicarbonyl-val-proarg-AMC),histidine-7AMC, $\alpha$-glutamic acid -7AMC, threonine-7AMC,lysine - 7AMC, phenylalanine-7AMC, proline7AMC,pyroglutamic acid-7AMC and tyrosine-7AMC (Dawne, 1993; Teresa Spanu species, 2008).

In addition the card includes a total of 16 fermentation tests (for amygdaline, arbutine, d-galactose, d-glucose, d-maltose, dmannitol, d-melibiose, d-raffinose, dsorbitol, d-trehalose, d-xylose, glycerol, 
lactose, l-arabinose, N-acetyl-glucosamine and salicin), two decarboxylase tests (for arginine and ornithine), and six other tests (for urease, pyruvate, optochin, novobiocin, polymyxin B sulfate, and $6.5 \% \mathrm{NaCl}$ ) (Teresa Spanu species, 2008).

Each organism suspension was prepared from the growth of pure cultures of bacteria cultivated on plates containing Trypticase soy agar with $5 \%$ sheep blood (bioMérieux, Marcy l'Etoile, France) and incubated overnight at $35^{\circ} \mathrm{C}$. Bacterial cells were suspended in $2.5 \mathrm{ml}$ of a $0.45 \%$ sodium chloride solution. The suspension used in the VITEK 2 system was adjusted to a McFarland standard of 0.5 by using a Densicheck (bioMérieux) (Teresa Spanu species, 2008).

In some cases, when the automated reading is inconclusive (i.e., low discrimination between two or more species), the manufacturer suggests one or more simple manual tests to resolve the identity of the test strain (i.e., hemolysis, coagulase tube test, clumping factor reaction, susceptibility to polymyxin $\mathrm{B}$, or colony pigmentation). All of these tests were carried out according to standard procedures (Teresa Spanu species, 2008).

\section{Results and Discussion}

Out of 60 isolates, $58 \%$ of the samples were from exudates, 33\% were from urine and $9 \%$ were from blood. All these isolates were speciated based on the conventional method as well as automated method for determining the sensitivity and efficacy of each method.

According to conventional method, out of 60 isolates Staphylococcus haemolyticus (26\%) was most frequently isolated, followed by S.cohnii (23\%), S.xylosus
(13\%), S.lugdunensis (11\%), S.epidermis (10\%), S.stimulans (10\%), S.saprophyticus (3\%), S. schleiferi (3\%) and S.warnerii (3\%). This finding was based on the conventional method of speciating CONS. From the total of 60 isolates Vitek correctly identified S.hamolyticus (26\%), S.cohnii(23\%), S.epidermidis (10\%) and S.saprophyticus (3\%), for the remaining species like S.xylosus, S.lugdunensis, S.simulans, S.schleiferi and S.warneri the result was ambiguous and correct identification was done based on the supplemental test suggested by the manufacturer, by the conventional method only $3 \%$ was misidentified to be S.warneri which the automated method identified to be S.epidermidis. The graphical representation of this comparative study is shown in figure 1 .

Antibiotic sensitivity testing of overall 60 isolates showed $36 \%$ of Methicillin resistant CONS(MRCONS) and $64 \%$ of Methicillin sensitive CONS. Ciprofloxacin was found to be sensitive in $61 \%$ isolates whereas resistant in $36 \%$ and intermediate was found to be $3 \%$ Cotrimaxazole was found resistant for $40 \%$ isolates and sensitive for $60 \%$.Among aminoglycoside group of drugs Gentamycin showed resistance to $7 \%$ isolates rest being sensitive, 67\% isolates were resistant to erythromycin (63\%) as compared to clindamycin which showed resistance of only 13\%.In between MRCONS and MSCONS maximum antibiotic resistance pattern was found in MRCONS. The difference pattern is shown in figure no. 2

Among the 60 isolates S.haemolyticus showed maximum resistance to methicillin followed by S.epidermidis, S.xylosus, S.simulans, S.saprophyticus and S.cohnii shown in figure 3. 
Nosocomial infections are major source of morbidity, mortality and also monetary burden on patients. Primary bloodstream infections, pneumonia, and urinary tract infections associated with invasive devices make up the great majority of nosocomial infections caused by staphylococcus species. The major cause of nosocomial infection is due to the long term irrational use of antimicrobials which leads to the development of resistant strains of pathogens (Clinical Laboratory Standard Institute Guidelines, 2014-2015).

Few organisms causing various nosocomial infections are Pseudomonas aeruginosa, Acinetobacter baumanii, vancomycin resistant Enterococcus, ventilator associated pneumonia by Staphylococcus aureus, Methicillin resistant Staphylococcus aureus etc. Among which nosocomial infection caused by Staphylococci species are important. Many laboratories do not identify CONS at species level as they were once considered simple commensal of human skin and mucous membrane but now are opportunistic pathogens. In laboratories the use of only screening tests leads to misidentification of the organism. The conventional method of Kloos and Schleifer is highly reliable and accurate but it is also time consuming and labor-intensive.

For this reasons automated commercial systems have been introduced for routine laboratory use. Most important advantage of these systems is the significant savings in terms of the time needed for species level identification. At present the new fluorescence based technology of the Vitek 2 system is more advanced and shows undoubted positive results.

Table.1

\begin{tabular}{|l|l|l|}
\hline \multicolumn{1}{|c|}{ SPECIES } & \multicolumn{1}{|c|}{ SUBSPECIES } & \multicolumn{1}{c|}{ NATURAL HOSTS } \\
\hline S.aureus & Aureus & Humans, mammals, birds \\
\hline S.epidermidis & & Humans \\
\hline S.capitis & Capitis & Humans \\
\hline S.capitis & Ureolyticus & Humans, some primates \\
\hline S.caprae & & Humans, goats \\
\hline S. saccharolyticus & & Humans \\
\hline S. warneri & & Humans, primates, domestic mammals. \\
\hline S.haemolyticus & & Humans, primates, domestic mammals \\
\hline S.hominis & & Humans \\
\hline S.lugdunensis & & Humans \\
\hline S.auricularis & & Humans, primates \\
\hline S.cohnii & Cohnii & Humans \\
\hline S.cohnii & Urealyticum & Humans, primates \\
\hline S. saprophyticus & & Humans, mammals \\
\hline S.xylosus & & Humans, mammals, birds \\
\hline S. simulans & & Humans, mammals \\
\hline S.intermedius & & Mammals, birds \\
\hline S. schleiferi & Humans \\
\hline
\end{tabular}


Table.2

\begin{tabular}{|l|l|l|}
\hline Screening tests & \multicolumn{1}{|c|}{ Primary tests } & \multicolumn{1}{c|}{ Confirmatory tests } \\
\hline Glucose & $\alpha$-Lactose & Esculin \\
Catalase & Nitrate reduction & Ornitine decarboxylase \\
Coagulase & Urea agar slant & Raffinose \\
(slide and & Arginine dihydrolase & D-Cellobiose \\
tube) & Acetoin (tube) & B-D-Fructose \\
Gram & Novobocin sensitivity & Galactose \\
staining & ß-Galactosidase & Arabinose \\
Colony size & & \\
Colony & & \\
pigment & & \\
& & \\
\hline
\end{tabular}

Table.3

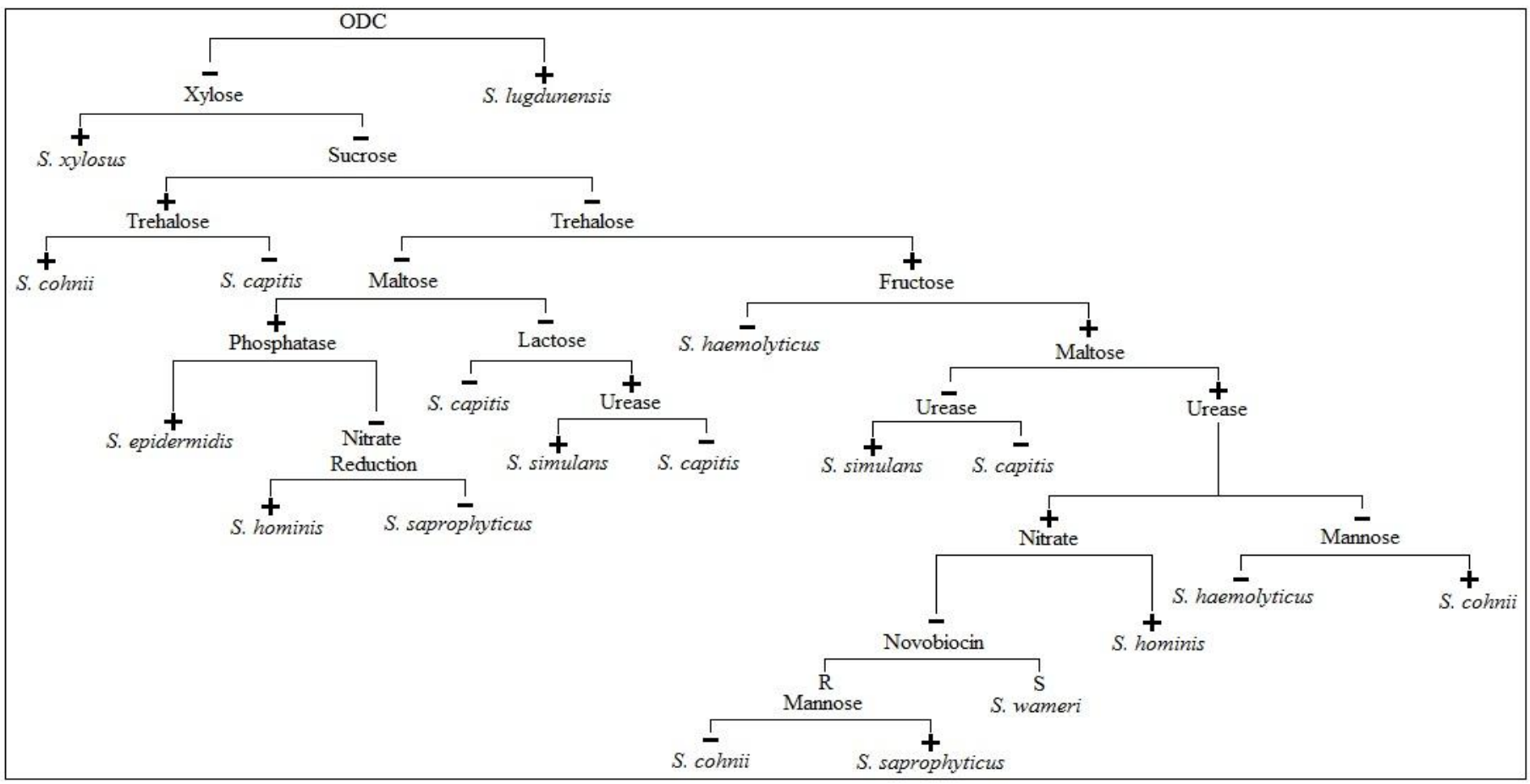



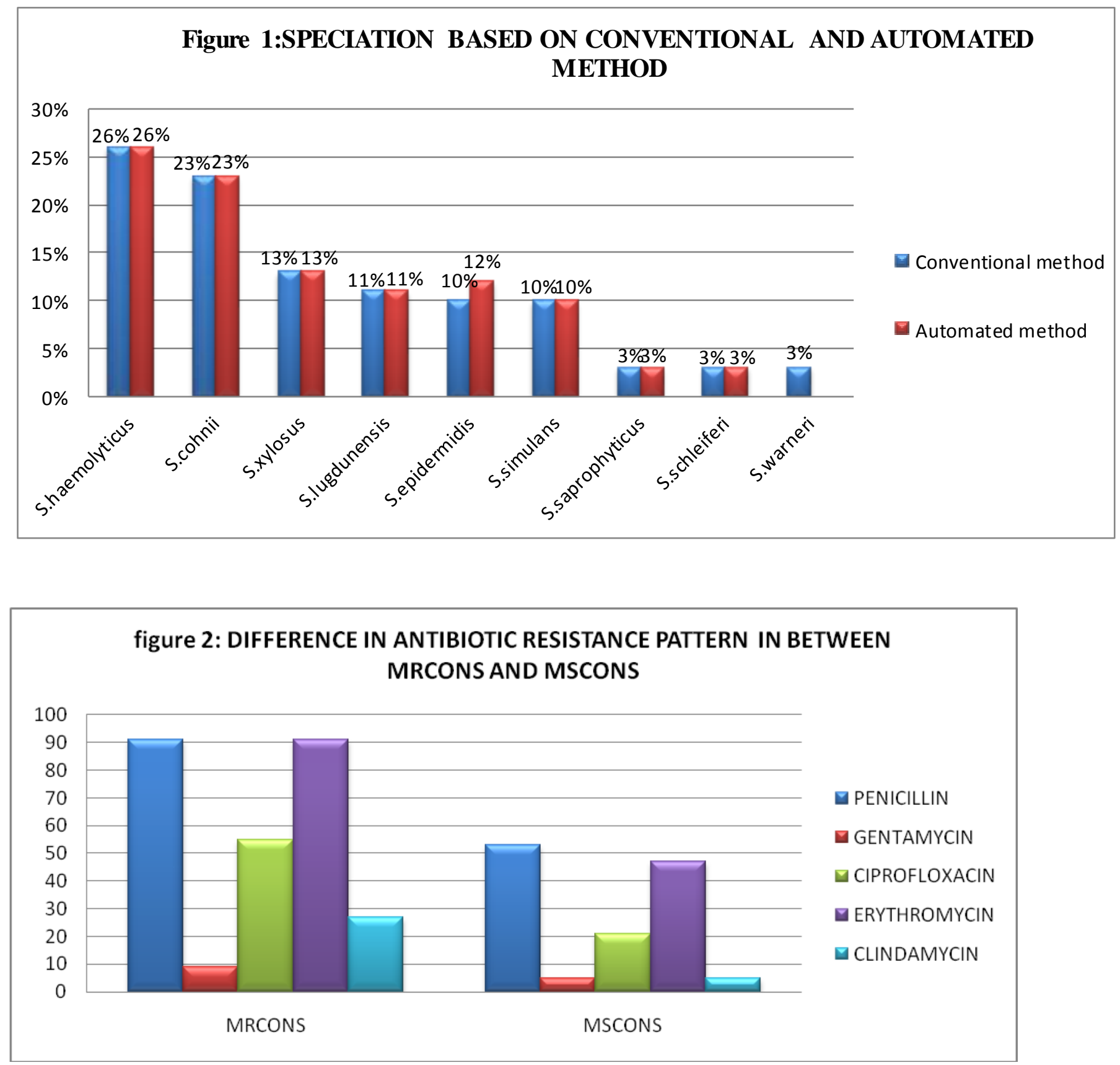


\section{figure 3 :PERCENTAGE OF METHICILLIN RESISTANCE IN DIFFERENT SPECIES OF CONS}

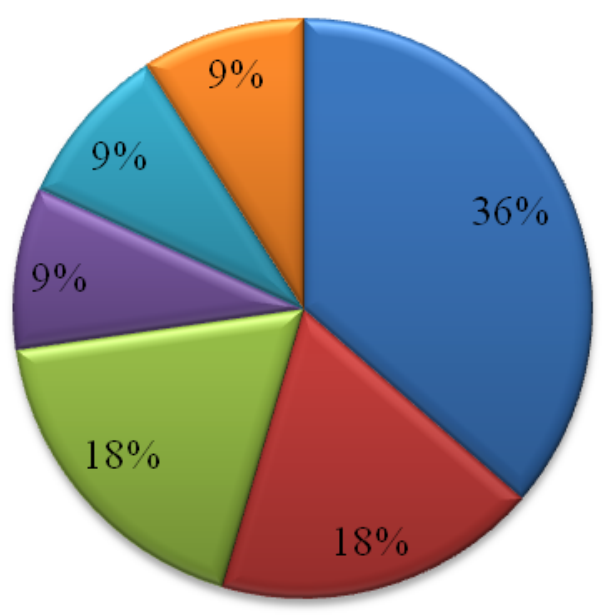

S.haemolyticus

$\square$ S.epidermidis

$\square$ S.xylosus

- S.simulans

$\square$ S.saprophyticus

$\square$ S.cohnii

The present scheme of Kloos and Schleifer conveniently identified the most frequently encountered clinical isolates in our hospital. Based on the conventional method biochemical characterization, the most frequently encountered species in our hospital was Staphylococcus haemolyticus (26\%) followed by S.cohnii (23\%), S.xylosus (13\%), S.lugdunensis (11\%), S.epidermis (10\%), S.simulans (10\%), S.saprophyticus (3\%), S.schleiferi (3\%) and S.warnerii (3\%) by the incorporation of few additional tests. According to a study done by jain, et al, S.haemolyticus and S.epidermidis were most commonly isolated. Similar kind of percentage of S.haemolyticus followed by S.saprophyticus, S.epidermidis was also seen in other studies by Manijeh et al.

According to the automated method done by Vitek 2 system, the result observed in my study was, from the total of 60 isolates of CONS, Vitek identified S.haemolyticus (26\%), S.cohnii(23\%), S.epidermidis(13\%) and S.saprophyticus (3\%), for the remaining species like S.xylosus, S.lugdunensis, S.simulans, S.schleiferi and S.warneri the result was ambiguous and correct identification was done based on the supplemental test suggested by the kit literature, by the conventional method only $3 \%$ was identified to be S.warneri which the automated method identified to be S.epidermidis similar type of results were also obtained in other studies by Teresa Spanu et al.

The antibiotic susceptibility pattern showed no resistance to vancomycin with high resistance to Penicillin, Cefazolin, Cotrimaxazole and Erythromycin same kind of result was seen in the study by Goyal et al., About $36 \%$ from the total isolates showed methicillin resitance. The species showing maximum resiatnce to methicillin was S.haemolyticus followed by S.epidermidis which was similar to the study by Subhra Singh et al., Penicillin, Gentamycin, Ciprofloxacin, Erythromycin and Clindamycin showed higher resistance in MRCONS isolates (Maazuddin et al., 2014).

CONS are becoming major cause of nosocomial infections and emergence of various drug resistances makes it important 
for adopting various methods for speciation of CONS and treating the infection caused by this group of organisms.

It is therefore recommended to assess the importance of CoNS, speciate the clinically relevant CoNS to whatever level possible and perform the antibiotic susceptibility testing before any typing procedure for epidemiological studies. This simple, inexpensive methodology will prove useful in a routine microbiology laboratory for the presumptive identification of CoNS. Further studies are needed with well characterized strains for the evaluation of the present schemes used in our laboratory.

In conclusion, the two methods used in our laboratory were found to be highly efficient for routine use due to their high sensitivity and specificity in addition to requiring fewer tests and thus being more economical and faster than the standard method.

\section{References}

Ciraj, M., P. Vinod, G. Sreejith, K. Rajani. 2009. Inducible clindamycin resistance among clinical isolates of staphylococci. Indian $J$. Pathol. Microbiol., Vol.: 52, No.:1.

Clinical Laboratory Standard Institute Guidelines. 2014-2015.

Dawne, L. 1993. Rodent. Numerical approach to reference identification of Staphylococcus, Stomatococcus and Micrococcus. J. Clin. Microbiol., Vol.: 31, No.: 3, Page no.: 490493.

Goyal, R. 2006. Simple and economical method for speciation and resistotyping of clinically significant coagulase negative staphylococci. Indian J. Med. Microbiol., Vol.: 24, No.: 3. Page no.: 201-204.

Julien Delmas, Jean Paul Chacornac. Evaluation of the Vitek 2 system with a variety of Staphylococcus Page no.: 311-313.

Konemann. Koneman's Color Atlas and Textbook of Diagnostic Microbiology $\left(6^{\text {th }}\right.$ edition). Lipincott Williams and Wilkins, Philadelphia. Page no.: 623.

Maazuddin Mohammed, Arshd H. Mohammed, Misba Ali, B., Mirza, Azizullah Ghori. 2014. Nosocomial infection - an overview. Int. Res. J. Pharm.

Maria de Lourdes, R.S., Cunha, Yuri, K., Sinzato, Liciana, V.A., Silveira. 2004. Comparison of Methods for the Identification of Coagulase-negative Staphylococci. Memórias do Instituto Oswaldo Cruz, Vol.: 99, No.: 8. Page no.: 855-860.

Maurizio Sanguinetti. 2003. Use of the Vitek 2 system for rapid identification of clinical isolates of Staphylococci from bloodstream infections. J. Clin. Microbiol., Vol.: 41, No.: 9. Page no.: 4259-4263.

Shubra Singh. 2008. Simple method for speciation of clinically significant coagulase negatives Staphylococci and its antibiotic sensitivity/resistant pattern in NICU of tertiary care center. Indian J. Med. Microbiol., Vol.: 19, No.: 2. Page no.: 0508.

Teresa Spanu species. 2008. J. Clin. Microbiol.Vol.: 46, No.: 1.

Wesley, E., Kloos, Jammy, L. 1994. Bannerman. Update of clinically significance of Coagulase Negative Staphylococci. Clin. Microbiol. Rev., Vol.: 7. Page no.: 117-140.

Wesley, E., Kloos, Karl, H., Schleifer. 1975. Simplified scheme for routine identification of human Staphylococcus species. J. Clin. Microbiol., Vol.: 1. Page no.: 82-88.

\section{How to cite this article:}

Umamagewari, S.S.M., P. Neelu Sree, Madhurima Mukerjee, M. Kalyani and Shameem banu, A.S. 2016. Comparative Study between Conventional Methods over Automated Methods for Speciation of Coagulase Negative Staphylococcus in a Tertiary Care Center. Int.J.Curr.Microbiol.App.Sci. 5(10): 639-647. doi: http://dx.doi.org/10.20546/ijcmas.2016.510.071 